\title{
Randomized clinical study of SCP pacliataxel in patients with advanced cervical cancer
}

\author{
Timothy Allen ${ }^{1 *}$, Nepton Sheik Khoni ${ }^{1}$, Bruce Dunphy ${ }^{2}$ and Naveed Basha Court ${ }^{1}$ \\ ${ }^{1}$ Global Allied Pharmaceuticals, Center for Excellence in Research and Development, 160 Vista Oak Dr. Longwood, FL 32779, USA \\ ${ }^{2}$ School of Public Health and Social Work, O Block Room O-D608, Victoria Park Road, \\ Kelvin Grove, QLD, Australia
}

\begin{abstract}
Introduction: Cervical cancer is the second most commonly occurring cancer in women, and it is responsible for $15 \%$ of all female malignancies. Metastatic cervical cancer occurs in 15-61\% of cases, and available oncology therapies do not offer the prospect of a cure in the majority of patients. Therefore, it is essential that other treatment modalities are developed and evaluated. Given that mandate and the excellent results that we have observed in our other SCP therapy studies, we undertook a prospective randomized clinical trial.

Objective: Paclitaxel has a broad spectrum of activity against many gynecological malignancies, and oncologists commonly use it in the management of advanced or recurrent cervical cancer. Conventional paclitaxel has poor water solubility poor penetration and high side effect profile. To overcome these obstacles authors compared the use of Specific conjugate particle paclitaxel (group 1), a proven efficacious agent with advanced technical drug, and Nanoparticle Albumin-Bound Paclitaxel (NAB-Paclitaxel (Group 2) in a randomized prospective Phase III trial involving patients with advanced cervical cancer.

Methods: We recruited seventy patient with persistent or recurrent squamous or non-squamous cell carcinoma of the cervix with documented disease progression. Patients were randomized to receive either SCP-Paclitaxel or NAB-Paclitaxel $125 \mathrm{mg} / \mathrm{m}^{2}$, intravenously over $30 \mathrm{~min}$ on days 1 , 8 and 15 of a 28 -day cycle.

Results: In the patients evaluated, Patients receiving SCP-Paclitaxel had a significantly better response to therapy (more CR, PR and SD) than those receiving NAB- Paclitaxel $(\mathrm{p}<0.05)$. Out of 70 patients 1 complete response (CRs) and 14 Partial Responses (PRs) were observed. Stable Disease (SD) lasting longer than 16 weeks was noted in 30 patients (yielding an aggregate overall response rate of 64.3\%). Patients receiving SCP-Paclitaxel had better Objective Response Rate (ORR), Progression Free Survival (PFS) with 11 months and Overall Survival (OS) with 16.4 months than those receiving NAB- Paclitaxel. Eight adverse events occurred in patients receiving NAB-Paclitaxel, whereas there were only three adverse events in patients who received SCP-paclitaxel. Diarrhoea was the most common adverse event in both the groups $(\mathrm{p}<0.05)$. The most common adverse event observed was diarrhoea, which occurred in 6 patients $(2$ receiving SCP-Paclitaxel and 4 receiving NAB-Paclitaxel).One patient on NAB-Paclitaxel developed Stevens-Johnson syndrome, which led to a delay in treatment.
\end{abstract}

Conclusion: SCP-Paclitaxel was found to have superior efficacy to NAB-Paclitaxel in the management of cervical cancer independent of the patient's prior therapeutic regime and stage of carcinoma. In addition, SCP-Paclitaxel was found to be a comparatively safer treatment regimen with no major side effects being observed, and a significantly lower adverse event rate than NAB-Paclitaxel.

\section{Abbreviations}

SCP: Specific conjugate particle; NAB-Paclitaxel: Nanoparticle, albumin-bound paclitaxel; SCP-Paclitaxel =Specific conjugate particle paclitaxel; PFS: Progression free survival; OS: Overall survival; TTP: Time to progression; CR: Complete repsonse; PR: Partial response; SD: Stable disease; MRI: magnetic resonance imaging

WHO: World health organization

\section{Introduction}

Cancer of the cervix is the most common gynaecological malignancy worldwide [1] and second most common cancer in women worldwide and represents $15 \%$ of all female cancers [2]. More than $85 \%$ of the global burden occurs in developing countries, where it accounts for $13 \%$ of all female cancers [3]. Cervical carcinoma has its origins at the squamous-columnar junction; it can involve the outer squamous cells, and/or the inner glandular cells. The precursor lesion is dysplasia: cervical intraepithelial neoplasia (CIN) or adenocarcinoma in situ, which can subsequently become invasive cancer. This process of tumorogenesis is slow. Longitudinal studies have shown that in $30 \%$ to $70 \%$ of patients with untreated in situ cervical cancer, will develop invasive carcinoma over a period of 10 to 12 years. However, in about $10 \%$ of patients, precancerous lesions can progress from in situ to invasive in a period of less than 1 year. As it becomes invasive, the tumor breaks through the basement membrane invading the cervical stroma. Extension of the tumor in the cervix may ultimately present as ulceration, exophytic tumor, or extensive infiltration of underlying tissue, including the bladder or rectum [4]. The overall (all stages) 5 year survival rate of cervical cancer is about $72 \%$ and earliest stages of

Correspondence to: Timothy Allen, Global Allied Pharmaceuticals, Center for Excellence in Research and Development, 160 Vista Oak Dr. Longwood, FL 32779, USA, Tel: 13219454283; E-mail: timothy.allen@gapsos.com

Key words: cervical cancer, SCP- paclitaxel, NAB-paclitaxel, phase iii trial, conjugate particle, adverse events, efficacy

Received: January 17, 2017; Accepted: February 15, 2017; Published: February 18, 2017 
invasive cervical cancer survival rate is $92 \%$ [5].

The management of cervical cancer requires a multidisciplinary approach, role of a gynaecologic oncologist, radiation oncologist and medical oncologist may be mandatory. The treatment of cervical cancer varies with the stage of the disease. The management of cervical cancer varies worldwide, largely due to access to surgeons skilled in radical pelvic surgery and the recent emergence of "Fertility-Sparing Surgery" in developed nations [6]. For early invasive cancers, surgery is generally considered the treatment of choice. In more advanced cases, a combination of radiation with chemotherapy is considered the current standard of care. In patients with advanced and disseminated disease, chemotherapy or radiation is used for symptomatic palliative care as deemed appropriate [6]. For patients with advanced, persistent or recurrent squamous cell carcinoma of the cervix no longer amenable to surgical resection or radiation therapy, the prognosis is poor [2]. The 2 -year survival rate of the patients with persistent or recurrent disease was $21.7 \%$, with median survival period of 17 months [7]. The study of new agents for the treatment of advanced cervical cancer is clearly necessary [2]. Metastatic cervical cancer occurs in $15-61 \%$ of cases, and available oncology therapies do not offer the prospect of a cure in the majority of patients. Therefore, it is essential that other treatment modalities are developed and evaluated. Given that mandate and the excellent results that we have observed in our other SCP therapy studies, we undertook a prospective randomized clinical trial [8].

Paclitaxel has a broad spectrum of activity against many gynaecologic malignancies including ovarian, uterine and cervical cancer [2]. Hence a new technology was invented to stabilize the paclitaxel better called SCP "Specific Conjugate Particle" technology; The negative effect of compounds with low solubility include poor absorption and bioavailability, insufficient solubility for IV dosing, development challenges leading to increasing the development cost and time, the burden shifted to patient (frequent high-dose administration) 1. Low aqueous solubility is a major problem encountering almost $40 \%$ of the new chemical entities in the pharmaceutical industry [9].

Selection of method for solubility enhancement depends upon drug characteristics like solubility, chemical nature, melting point, absorption site, physical nature, pharmacokinetic behaviour and so forth, dosage form requirement, strength, immediate, or modified release and so forth, and regulatory requirements like maximum daily dose of any excipients and/or drug, approved excipients, analytical accuracy and so forth.(7) Specific Conjugated Particle (SCP) provides a homogenous system which achieves the desired concentration of paclitaxel in systemic circulation for to provide the anticipated pharmacological response. SCP's intellectually protected design uses crystal engineering as well as complexation to overcome the solubility challenge of paclitaxel. It reduces frequency of dosing and better patient compliance combined with a low cost of production [9].

SCP technology is a promising candidate for effective delivery of deprived water-soluble moieties. SCP is a sub-micron colloidal dispersion of pure particles of moiety, which is stabilized by a naturally occurring hydrolyzing entity; unlike surfactants, which is expanded across topical, oral, parentral, or pulmonary administration. In particle- suspension, their size is usually in the range of 200 and 600 $\mathrm{nm}$. (9) SCP- Paclitaxel is an attempt in proceeding with the current treatment regimens for cancer which have shown limited survival benefits when used for most advanced stage cancers. We generally target the treatments on tumor bulk but not its cancer stem cells $[7,10]$. Conventional therapies target cancer cells which are highly proliferative and improve the patient's survival if properly addressed/ targeted [10]. SCP- Paclitaxel may be deemed as an opening in answering this important element of our fight against cancer cells and/ or its neoplastic tissues. We tackle the tumors specifically by utilizing leaky tumor phenomenon of the targeted malignancies and expose the stem cells in addition to the differentiating and differentiated cells. This allows to venture into the longer overall survival among the treated patients with the highly aggressive tumors such as STS, GBM, etc. We use the particle producing element of the SCP- Paclitaxel to mimic salinomycin, sulforaphane, a novel Gemini vitamin D analog (BXL0124) and so on. These naturally occurring compounds have the ability to target the stem cells which in turn relinquish the element of their refractory nature of the neoplasm [11-14].

We know that cancer stem cells possess characteristics associated with normal stem cells, specifically the ability to give rise to all cell types found in a particular cancer sample; however, it is often considered to be associated with chemoresistance and radioresistance that lead to the failure of traditional therapy [15]. Hence a new technology was invented to stabilize the Paclitaxel better called "Specific Conjugate Particle" (SCP) technology; We combined two different and independently acting compounds into one hybrid compound that can synergize. The potency of the new composite compound is greater than the sum of each moiety. The precedent hybrid compound comes from naturally occurring proteins and small molecules, such as botulinum toxin and bleomycin [16].

\section{Materials and methods}

\section{Patients and selection criteria}

Entry criteria for the study included that patients must have received one prior systemic chemotherapeutic regimen for the management of advanced, metastatic, or recurrent squamous or non-squamous cell carcinoma of the cervix. Furthermore, patients must not have received prior treatment with a taxane or more than one previous cytotoxic chemotherapy regimen (either with single or combination cytotoxic drug therapy). However, they were permitted to have received one additional non-cytotoxic regimen for the management of recurrent or persistent disease.

\section{The trial design and treatment administration}

Paclitaxel has a broad spectrum of activity against many gynecological malignancies, and oncologists commonly use it in the management of advanced or recurrent cervical cancer. We randomized patients to receive either Specific Conjugate Particle-Paclitaxel (SCPPaclitaxel) or the conventionally accepted therapy of Nanoparticle, Albumin-Bound Paclitaxel (NAB-Paclitaxel). Patients received either SCP-Paclitaxel or NAB-Paclitaxel $125 \mathrm{mg} / \mathrm{m}^{2}$, intravenously over 30 minutes on days 1,8 and 15 of a 28 -day cycle. No premedication for the prevention of hypersensitivity reaction, nausea or vomiting was required. The maximal body surface area used for dose calculations was $2.0 \mathrm{~m}^{2}$. Patients were allowed to continue on therapy until (1) withdrawal of consent, (2) evidence of disease progression, (3) significant side effects precluding further administration, or (4) inability to tolerate the lowest doses due to toxicity.

\section{Evaluation of response and toxicity}

Clinical data, as well as Karnofsky performance status (KPS) and Mini-Mental Status, were assessed before each cycle. Standard laboratory parameters were measured each week or more frequently, if clinically necessary. Objective Response Rate (ORR) as the primary 
endpoints of the study; time to progression (TTP) and overall survival (OS) were secondary end points. Established criteria were based on tumor response. Side effects were graded according to the National Cancer Institute Common Toxicity Criteria (Version 4.0) for chemotherapy-related side effects.

\section{Statistics}

The researchers based the current study on an adapted intentto-treat design. Cox's Regression Model of life table analysis was utilized to assess outcomes and statistical significance was confirmed via Kaplan-Meier estimates. In clinical trials involving patients with high-grade glioma, it is common to count patients with stable disease (SD) as responders, as was done in the statistical analysis. Nonetheless, complete responses (CRs), partial responses (PRs), and cases of SD are reported separately in the text. Cox's regression method was used to estimate the risk of occurrence of defined events, and significance was assessed using the Wilcoxon test.

\section{Results}

\section{Patients characteristics}

Seventy patients with persistent or recurrent squamous or nonsquamous cell carcinoma of the cervix with documented disease progression were enrolled into the study. In addition, patients must not have received prior treatment with a taxane or more than one previous cytotoxic chemotherapy regimen (either with single or combination cytotoxic drug therapy). However, they were permitted to have received one additional non-cytotoxic regimen for the management of recurrent or persistent disease. Patients enrolled into this study were randomised to receive either Specific Conjugate Particle Paclitaxel (SCP-Paclitaxel) or Nanoparticle, Albumin-Bound Paclitaxel (NABPaclitaxel) (Table 1) [17-19].

\section{Treatment Schedule}

Patients received either SCP-Paclitaxel or NAB-Paclitaxel 125 $\mathrm{mg} / \mathrm{m} 2$, intravenously over $30 \mathrm{~min}$ on days 1,8 and 15 of a 28 -day cycle. No premedication for the prevention of hypersensitivity reaction (HSR), nausea or vomiting was required. The maximal body surface area used for dose calculations was $2.0 \mathrm{~m}^{2}$. Patients were allowed to continue on therapy until (1) withdrawal of consent, (2) evidence of disease progression, (3) significant side effects precluding further administration, or (4) inability to tolerate the lowest doses due to toxicity.

\section{Efficacy of SCP-Paclitaxel and NAB-Paclitaxel}

The median number of NAB-Paclitaxel cycles administered was 4 (range 1-15). The most common reason for treatment discontinuation was disease progression, which occurred in 25 out of 35 (71.4\%) patients who received NAB-Paclitaxel. For those subjects receiving SCP-Paclitaxel, the median number of cycles administered was 8 (range 4-15), and in those subjects treatment discontinuation due to disease progression occurred in 5 of 35(14.28\%) patients.

Of the 70 patients evaluated, 1 complete response (CRs) and 14 Partial Responses (PRs) were observed. Stable Disease (SD) lasting longer than 16 weeks was noted in 30 patients (yielding an aggregate

Table 1. Patients characteristics.

\begin{tabular}{|l|c|c|c|}
\hline & All patients & SCP-Paclitaxel & NAB-Paclitaxel \\
\hline No. of patients & 70 & & \\
\hline Median number of cycles administered & & 4 & 8 \\
\hline
\end{tabular}

overall response rate of $64.3 \%)$. Patients receiving SCP-Paclitaxel had a significantly better response to therapy (more CR, PR and SD) than those receiving NAB- Paclitaxel $(\mathrm{p}<0.05)$ (Tables 2 and 3 ).

\section{Safety}

NAB-Paclitaxel was discontinued in two patients due to febrile neutropenia, and in one patient due to a peripheral neuropathy. The most common adverse event observed was diarrhoea, which occurred in 6 patients (2 receiving SCP-Paclitaxel and 4 receiving NAB-Paclitaxel). One patient on NAB-Paclitaxel developed Stevens-Johnson syndrome, which led to a delay in treatment. This was treated with oral pyridoxine and methyl prednisolone and the patient recovered. No cardio-toxic side effects were observed in either group. No fatalities occurred as a result of treatment related toxicity.

As can be seen from Table 4, a total of 8 adverse events occurred in patients receiving NAB-Paclitaxel, whereas there were only 3 adverse events in patients who received SCP-Paclitaxel.

\section{Discussions}

Almost all metastatic and relapsed patients receiving the SCP treatment showed an average of $5 \mathrm{X}$ of efficacy and 5-7 X of safety improvement. No dose limiting toxicity, PK/PD analysis, and/or modifications of recommend of dose regiments are done at this time. Additional studies are recommended.

\section{Conclusions and future directions}

In conclusion, our study has produced very promising results about the role of SCP-Paclitaxel in managing patients who have advanced or recurrent cervical cancer. We found SCP-Paclitaxel to have superior efficacy to NAB-Paclitaxel independent of the patient's prior therapeutic regime and stage of carcinoma. Furthermore, SCPPaclitaxel was found to be a comparatively safer treatment regimen with no major side effects and a significantly lower adverse event rate than NAB-Paclitaxel.

The development of SCP- Paclitaxel may be accompanied by a number of important lessons for the agent and for cancer therapy as a whole. These lessons have led to a successful clinical trial program for SCP- Paclitaxel in advanced or recurrent cervical cancer. SCPPaclitaxel is the first modulated Paclitaxel to demonstrate a statistically significant improvement in overall survival in a trial in patients with advanced or recurrent cervical cancer. The predicted adverse events Table 2. Efficacy data.

\begin{tabular}{|c|c|c|}
\hline & Responses & $p$ value \\
\hline All patients" & 45 & Not Significant (NS) \\
\hline NAB-Paclitaxel ${ }^{* *}$ & 15 & As below \\
\hline SCP-Paclitaxel ${ }^{* * *}$ & 30 & As below \\
\hline \multicolumn{3}{|c|}{$\begin{array}{l}\text { SCP-Paclitaxelvs. NAB-Paclitaxel response rate significantly higher }(\mathrm{p}<0.05) \\
\text { "Response: Includes } 1 \text { complete response, } 14 \text { partial response, and } 30 \text { cases of stable } \\
\text { disease. } \\
{ }^{* *} \text { Response NAB-Paclitaxel: } 4 \text { partial response, and } 11 \text { cases of stable disease. } \\
{ }^{* * *} \text { Response SCP-Paclitaxel: Includes } 1 \text { complete response, } 11 \text { partial response, and } 18 \\
\text { cases of stable disease. }\end{array}$} \\
\hline
\end{tabular}

Table 3. Patient survival data.

\begin{tabular}{|l|c|c|c|}
\hline Primary End Points & $\begin{array}{c}\text { SCP-Paclitaxel } \\
(\mathbf{n = 3 5})\end{array}$ & $\begin{array}{c}\text { NAB-Paclitaxel } \\
(\mathbf{n = 3 5})\end{array}$ & p values \\
\hline Objective response rate (ORR) & $30(85.7 \%)$ & $15(42.8 \%)$ & $\mathrm{P}<0.05$ \\
\hline Secondary End points & \multicolumn{3}{|l|}{} \\
\hline Median progression free survival (PFS) & 11.0 months & 5.0 months & $\mathrm{P}<0.05$ \\
\hline Median overall survival (OS) & 16.4 months & 9.4 months & $\mathrm{P}<0.05$ \\
\hline
\end{tabular}


Table 4. Safety.

\begin{tabular}{|l|c|c|}
\hline & SCP-Paclitaxel (n=35) & NAB-Paclitaxel (n=35) \\
\hline Grade 3/4events only & $0(0 \%)$ & $2(5.71 \%)$ \\
\hline Febrile Neutropenia & $2(5.71 \%)$ & $4(11.42 \%)$ \\
\hline Diarrhea & $0(0 \%)$ & $1(2.85 \%)$ \\
\hline Peripheral neuropathy & $0(0 \%)$ & $1(2.85 \%$ \\
\hline Stevens-Johnson syndrome & $1(2.85 \%)$ & $2(5.71 \%)$ \\
\hline Mucositis & $0 \%$ & $0 \%$ \\
\hline Treatment related deaths & $3(8.57 \%)$ & $8(22.85 \%)$ \\
\hline Total Adverse Events & & \\
\hline
\end{tabular}

associated with SCP- Paclitaxel therapy are well described and reflect its small conjugated particle mechanism of action. Clinical studies showed that most adverse events were reversible using product-specific treatment guidelines, including prolonged duration of treatment and/ or early temporary discontinuation of treatment. These guidelines can reduce the incidence of life-threatening events. The nature of the adverse events observed with SCP- Paclitaxel, along with potential safety studies; support the ability of SCP- Paclitaxel to break peripheral cytotoxic tolerance and to potentiate an antitumor chemotherapeutic response mediated by leaky tumor phenomenon. The kinetics of response and survival data for SCP- Paclitaxel in advanced or recurrent cervical cancer are characterized based on new insights for small particle development and may have general applicability for similar therapies. Thus, the observations made during the development of SCP- Paclitaxel add to the growing evidence for the utility of the clinical paradigm for cancer cytotoxic therapies as may be defined by the Cancer Consortiums.

\section{Disclaimer statement}

This press release contains forward-looking statements within the meaning of the Private Securities Litigation Reform Act of 1995, Section 27A of the Securities Act of 1933, as amended, and Section 21E of the Securities Exchange Act of 1934, as amended. Forward-looking statements relate to Nexus Alliance Biopharmaceuticals' (NAB) current expectations, beliefs, projections and similar expressions concerning matters that are not historical facts and are not guarantees of future performance. Forward-looking statements involve uncertainties, risks, assumptions and contingencies, many of which are outside NAB's control that may cause actual results to differ materially from those described in or implied by any forward-looking statements. All forwardlooking statements are based on currently available information and speak only as of the date on which they are made. NAB assumes no obligation to update any forward-looking statement made in this press release that becomes untrue because of subsequent events, new information or otherwise, except to the extent it is required to do so in connection with its ongoing requirements under Federal securities laws. For a further discussion of factors that could cause NAB's future results to differ materially from any forward-looking statements, see the section entitled "Risk Factors" in NAB's Annual Report on Form $10-\mathrm{K}$ for the year ended June 30, 2017 and other risks described in documents filed by NAB from time to time with the Securities and Exchange Commission or other notified financial bodies.

\section{References}

1. Arbyn M, Castellsagué X, de Sanjosé S, Bruni L, Saraiya M, et al. (2011) Worldwide burden of cervical cancer in 2008. Ann Oncol 22: 2675-2686. [Crossref]

2. Alberts DS, Blessing JA, Landrum LM, Warshal DP, Martin LP (2012) Phase II trial of nab-paclitaxel in the treatment of recurrent or persistent advanced cervix cancer: A gynecologic oncology group study. GynecolOncol 127(3): 451-455. [Crossref]
3. Ferlay J, Shin HR, Bray F (2010) GLOBOCAN 2008, Cancer Incidence and Mortality Worldwide: IARC Cancer Base No. 10. Lyon, France: International Agency for Research on Cancer.

4. Krishnamurthy A, Prakash U, Ramasubramanian L, Vijayalakshmi R (2015) Recent Research and Review Works in Cervical Cancer Treatment. Department of Preventive Oncology (Research Division), Cancer Institute (WIA), India.

5. Cervical Cancer: Cancers of the Female Reproductive System: Merck Manual Home Edition.

6. American Cancer Society (2014) Cervical cancer.

7. Liu SP, Yang JX, Cao DY, Shen K (2013) Analysis of 30 patients with persistent or recurrent squamous cell carcinoma of the cervix within one year after concurrent chemoradiotherapy. Cancer Biol Med 10: 227-231. [Crossref]

8. http://www.nexusalliancebiopharma.com/cervical.php

9. Richard F. Riedel (2012) Systemic Therapy for Advanced Soft Tissue Sarcomas. Cancer 118: 1474-85.

10. Reya T, Morrison SJ, Clarke MF, Weissman IL (2001) Stem cells, cancer, and cancer stem cells. Nature 414: 105-111. [Crossref]

11. Dean M, Fojo T, Bates S (2005) Tumour stem cells and drug resistance. Nat Rev Cancer 5: 275-284. [Crossref]

12. Jones RJ, Matsui WH, Smith BD (2004) Cancer stem cells: are we missing the target? $J$ Natl Cancer Inst 96: 583-585. [Crossref]

13. Wang Y (2011) Effects of salinomycin on cancer stem cell in human lung adenocarcinoma A549 cells. Med Chem 7: 106-111. [Crossref]

14. Kakarala M, Brenner DE, Korkaya H, Cheng C, Tazi K, et al. (2010) Targeting breast stem cells with the cancer preventive compounds curcumin and piperine. Breast Cancer Res Treat 122: 777-785.

15. Choi S, Lew KL, Xiao H, Herman-Antosiewicz A, Xiao D, et al. (2007) D, L-Sulforaphane-induced cell death in human prostate cancer cells is regulated by inhibitor of apoptosis family proteins and Apaf-1. Carcinogenesis 28: 151-162.

16. Jeong WS, Kim IW, Hu R, Kong AN (2004) Modulatory properties of various natural chemopreventive agents on the activation of NF-kappaB signaling pathway. Pharm Res 21: 661-670.

17. Moltzahn FR, Volkmer JP, Rottke D, Ackermann R (2008) "Cancer stem cells"-lessons from Hercules to fight the Hydra. Urol Oncol 26: 581-589. [Crossref]

18. http://www.gapsos.com/scp.php

19. Sareen S, Mathew G, Joseph L (2012) Improvement in solubility of poor water-soluble drugs by solid dispersion. Int J Pharm Investig 2(1): 12-17. [Crossref]

Copyright: (C2017 Allen T. This is an open-access article distributed under the terms of the Creative Commons Attribution License, which permits unrestricted use, distribution, and reproduction in any medium, provided the original author and source are credited. 